\title{
Association between betel-nut chewing and chronic kidney disease in men
}

\author{
Che-Yi Chou', Shi-Yann Cheng ${ }^{2}$, Jiung-Hsiun Liu', Wen-Chun Cheng ${ }^{3, *}$, I-Min Kang ${ }^{4}$, \\ Yu-Hsiang Tseng ${ }^{3}$, Chuen-Ming Shih ${ }^{3}$ and Walter $\mathrm{Chen}^{3}$ \\ 'Division of Nephrology, Department of Internal Medicine, China Medical University Hospital, Taichung, Taiwan, \\ Republic of China: ${ }^{2}$ Obstetrics and Gynecology, China Medical University Beigang Hospital, Taiwan, Republic \\ of China: ${ }^{3}$ Department of Internal Medicine, China Medical University Beigang Hospital, No. 123 Sinde Road, \\ Beigang Township, Yunlin County, 65152 Taiwan, Republic of China: ${ }^{4}$ Department of Family Medicine, \\ China Medical University Beigang Hospital, Taiwan, Republic of China
}

Submitted 3 April 2007: Accepted 19 May 2008: First published online 23 July 2008

\begin{abstract}
Background: Betel-nut use is associated with metabolic syndrome and obesity. However, the association between betel-nut chewing and risk for chronic kidney disease (CKD) is unknown. The present study was conducted to determine the association between betel-nut chewing and CKD in men.

Methods: We retrospectively reviewed health-check records of 3264 men in a hospital-based cross-sectional screening programme from 2003 to 2006. CKD was defined as estimated glomerular filtration rate less than $60 \mathrm{ml} / \mathrm{min} / 1 \cdot 73 \mathrm{~m}^{2}$ calculated by the Modification of Diet in Renal Disease formula. Risk factors for CKD including diabetes, hypertension, BMI, smoking, alcohol consumption and age were also considered.

Results: A total of $677(20 \cdot 7 \%)$ men were found to have CKD and $427(13 \cdot 1 \%)$ participants reported a history of betel-nut use. The prevalence $(24 \cdot 8 \%)$ of CKD in betel-nut users was significantly higher than that (11.3\%) of participants without betel-nut use $(P=0 \cdot 026)$. In multivariate logistic regression analysis with adjustments for age, hypertension, diabetes and hyperlipidaemia, betel-nut use was independently associated with CKD $(P<0 \cdot 001)$. The adjusted odds ratio for betel-nut use was $2 \cdot 572$ (95\% CI 1.917, 3.451).

Conclusions: Betel-nut use is associated with CKD in men. The association between betel-nut use and CKD is independent of age, BMI, smoking, alcohol consumption, hypertension, diabetes and hyperlipidaemia.
\end{abstract}

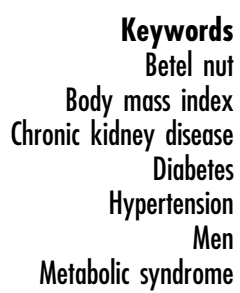

The incidence of end-stage renal disease (ESRD) is high and growing rapidly in adults younger than 65 years of age in Taiwan ${ }^{(1)}$. Outcomes of ESRD treatment tend to be poor; however, the cost of treatment is relatively high ${ }^{(2,3)}$. Early detection of possible risk factors and early treatment of chronic kidney disease (CKD) may not only slow the decline of renal function, but also prevent the development of severe cardiovascular complications ${ }^{(4)}$. Apart from well-known risk factors for CKD including age, hypertension, diabetes, obesity and metabolic syndrome, the identification of other possible risk factors associated with CKD is a key for early detection and treatment as well. The prevalence of betel-nut use is about $10 \%$, with about 600 million users all over the world ${ }^{(5,6)}$, and most betel-nut chewers in Taiwan are male. The prevalence of betel-nut use is rising gradually in Taiwan, especially in rural areas ${ }^{(5)}$. Evidence has shown that betel-nut chewing is associated with oral cancer ${ }^{(7)}$, hyperglycaemia, obesity, metabolic syndrome ${ }^{(8)}$ and increase in urinary albumin excretion $^{(9)}$. Betel-nut chewing may be a risk factor for CKD. However, the association between betel-nut chewing and CKD is unknown. We conducted the present crosssectional retrospective study to determine the association between betel-nut chewing and CKD in Taiwanese men.

\section{Methods}

A total of 8027 (3264 men and 4763 women) records in a general health-check programme in China Medical University Beigang Hospital, Yunlin County, Taiwan, were reviewed from 2003 to 2006. The prevalence of betel-nut use (as chews of fresh Areca catechu nuts with Piper betle leaves and lime) was $13 \cdot 8 \%$ in men and $0.8 \%$ in women. In view of the lower prevalence of betel-nut use in women, data analysis was limited to 3264 men. CKD was 
defined as estimated glomerular filtration rate (eGFR) of less than $60 \mathrm{ml} / \mathrm{min} / 1.73 \mathrm{~m}^{2}$ calculated by the Modification of Diet in Renal Disease formula ${ }^{(4)}$. Basic data on the participants included age, body height, body weight, systolic blood pressure (SBP) and diastolic blood pressure (DBP). Hb, platelet count, blood urea nitrogen (BUN), creatinine, uric acid, aspartate aminotransferase (AST), alanine aminotransferase (ALT), albumin, globulin, cholesterol, TAG and fasting blood sugar were measured standard automated technology. Participants fasted for $12 \mathrm{~h}$ overnight before blood sampling in the morning. Data on lifestyle factors and habits were obtained using a questionnaire based on the National Health Institute of Taiwan standard health-check format ${ }^{(10)}$. Hypertension was defined as blood pressure of at least $140 / 90 \mathrm{mmHg}$ or the use of antihypertensive medication ${ }^{(11)}$. Diabetes mellitus was defined as fasting blood glucose level of $140 \mathrm{mg} / \mathrm{dl}$, non-fasting glucose of $200 \mathrm{mg} / \mathrm{dl}$ or a history of treatment for diabetes ${ }^{(12)}$. Smoking was defined as a history of smoking for more than 2 pack-years ${ }^{(13)}$. Alcohol consumption was defined in those people drinking at least one drink daily ${ }^{(14)}$. Hyperlipidaemia was defined as serum cholesterol level $\geq 200 \mathrm{mg} / \mathrm{dl}$, serum TAG $\geq 200 \mathrm{mg} / \mathrm{dl}$ or if lipid-lowering agents were being used. Betel chewing was categorized as never, sometimes or frequently in the last 6 months.

\section{Statistical analyses}

Data are reported as mean and standard deviation or frequency and percentage, as appropriate. Baseline characteristics and anthropometric factors were compared between patients with CKD and those without by Student's $t$ test or the Mann-Whitney $U$ test (as appropriate) for continuous variables and by the $\chi^{2}$ test for categorical variables. Logistic regression models were used to estimate the odds ratios for CKD. To determine associations between betel-nut use and CKD, a multivariate logistic regression model with adjustments for age, sex, hypertension and diabetes was used. $P<0.05$ was considered statistically significant. All calculations were carried out using the SPSS for Windows statistical software package version 12 (SPSS Inc., Chicago, IL, USA).

\section{Results}

A total of $677(20 \cdot 7 \%)$ participants were found to have CKD and $427(13 \cdot 1 \%)$ participants had a history of

Table 1 Demographic and biochemical data of study participants by chronic kidney disease (CKD) status: retrospective review of healthcheck records of 3264 men in a hospital-based cross-sectional screening programme, Yunlin County, Taiwan, 2003 to 2006

\begin{tabular}{|c|c|c|c|c|c|c|}
\hline & \multicolumn{2}{|c|}{ Overall } & \multicolumn{2}{|c|}{$\begin{array}{l}\text { CKD }(-) \\
(n 2587)\end{array}$} & \multicolumn{2}{|c|}{$\begin{array}{c}\mathrm{CKD}(+) \\
(n 677)\end{array}$} \\
\hline & Mean & SD & Mean & SD & Mean & SD \\
\hline Age (years) & $64 \cdot 5$ & $11 \cdot 6$ & $62 \cdot 7$ & $11 \cdot 6$ & $71 \cdot 7^{\star}$ & $8 \cdot 3$ \\
\hline eĞFR $\left(\mathrm{ml} / \mathrm{min} / 1.73 \mathrm{~m}^{2}\right)$ & $74 \cdot 5$ & $19 \cdot 6$ & $81 \cdot 0$ & $16 \cdot 0$ & $49 \cdot 0^{*}$ & $11 \cdot 0$ \\
\hline BMI $\left(\mathrm{kg} / \mathrm{m}^{2}\right)$ & $24 \cdot 8$ & $3 \cdot 3$ & $24 \cdot 8$ & $3 \cdot 4$ & $24 \cdot 5^{\star}$ & $3 \cdot 2$ \\
\hline Height (cm) & $164 \cdot 1$ & $6 \cdot 4$ & $164 \cdot 2$ & $6 \cdot 5$ & $163 \cdot 9$ & $5 \cdot 9$ \\
\hline Weight (kg) & $66 \cdot 8$ & $10 \cdot 4$ & $67 \cdot 0$ & $10 \cdot 4$ & $66 \cdot 0^{*}$ & $10 \cdot 0$ \\
\hline SBP $(\mathrm{mmHg})$ & 134 & 23 & 132 & 22 & $141^{*}$ & 24 \\
\hline $\mathrm{DBP}(\mathrm{mmHg})$ & 78 & 13 & 78 & 12 & 79 & 14 \\
\hline $\mathrm{Hb}(\mathrm{g} / \mathrm{dl})$ & $14 \cdot 6$ & $1 \cdot 4$ & $14 \cdot 7$ & $1 \cdot 3$ & $14 \cdot 0^{*}$ & $1 \cdot 7$ \\
\hline Platelets $\left(10^{3} / \mu \mathrm{l}\right)$ & 199 & 56 & 200 & 56 & 191 & 55 \\
\hline AST (IU/I) & 34 & 29 & 34 & 31 & 33 & 22 \\
\hline ALT (IU/I) & 35 & 49 & 36 & 53 & $32^{*}$ & 33 \\
\hline BUN (mg/dl) & 18 & 7 & 17 & 5 & $24^{\star}$ & 10 \\
\hline Creatinine (mg/dl) & $1 \cdot 1$ & $0 \cdot 4$ & $1 \cdot 0$ & $0 \cdot 1$ & $1 \cdot 6^{*}$ & $0 \cdot 7$ \\
\hline Uric acid (mg/dl) & $6 \cdot 5$ & $1 \cdot 5$ & $6 \cdot 3$ & $1 \cdot 4$ & $7 \cdot 4^{*}$ & $1 \cdot 6$ \\
\hline Albumin (g/dl) & $4 \cdot 0$ & $0 \cdot 3$ & $4 \cdot 0$ & $0 \cdot 3$ & $3 \cdot 9^{*}$ & $0 \cdot 4$ \\
\hline Globulin (g/dl) & $3 \cdot 2$ & 0.5 & $3 \cdot 2$ & $0 \cdot 5$ & $3 \cdot 3$ & 0.5 \\
\hline Cholesterol (mg/dl) & 194 & 39 & 194 & 40 & 195 & 40 \\
\hline TAG (mg/dl) & 129 & 138 & 129 & 143 & 129 & 82 \\
\hline \multirow[t]{2}{*}{ Fasting blood glucose (mg/dl) } & 113 & 46 & 113 & 46 & 111 & 45 \\
\hline & $n$ & $\%$ & $n$ & $\%$ & $n$ & $\%$ \\
\hline \multicolumn{7}{|l|}{ Co-morbidity } \\
\hline Hypertension & 749 & $22 \cdot 9$ & 501 & $19 \cdot 4$ & 248 & $36 \cdot 6^{*}$ \\
\hline Diabetes & 406 & $12 \cdot 4$ & 304 & $11 \cdot 5$ & 102 & $15 \cdot 1^{*}$ \\
\hline Hyperlipidaemia & 127 & 3.9 & 101 & 3.9 & 26 & $3 \cdot 8$ \\
\hline \multicolumn{7}{|l|}{ Lifestyle } \\
\hline Smoking & 855 & $26 \cdot 2$ & 792 & $30 \cdot 6$ & 166 & $24 \cdot 5^{\star}$ \\
\hline Alcohol consumption & 958 & $29 \cdot 4$ & 746 & $28 \cdot 8$ & 109 & $16 \cdot 1^{*}$ \\
\hline Betel-nut use & 427 & $13 \cdot 1$ & 321 & $12 \cdot 4$ & 106 & $15 \cdot 7^{*}$ \\
\hline
\end{tabular}

eGFR, estimated glomerular filtration rate by Modification of Diet in Renal Disease formula; SBP, systolic blood pressure; DBP, diastolic blood pressure; AST, aspartate aminotransferase; ALT, alanine aminotransferase; BUN, blood urea nitrogen.

${ }^{\star} P<0.05$ v. CKD $(-)$ in independent $t$ test, Mann-Whitney $U$ test or $\chi^{2}$ test. 
Table 2 Demographic and biochemical data of participants by history of betel-nut use: retrospective review of health-check records of 3264 men in a hospital-based cross-sectional screening programme, Yunlin County, Taiwan, 2003 to 2006

\begin{tabular}{|c|c|c|c|c|}
\hline & \multicolumn{2}{|c|}{$\begin{array}{l}\text { Betel nut(-) } \\
\quad(n 2837)\end{array}$} & \multicolumn{2}{|c|}{$\begin{array}{l}\text { Betel nut(+) } \\
\quad(n \text { 427) }\end{array}$} \\
\hline & Mean & SD & Mean & SD \\
\hline Age (years) & $65 \cdot 2$ & $11 \cdot 3$ & $59 \cdot 8^{*}$ & $12 \cdot 2$ \\
\hline eGFR $\left(\mathrm{ml} / \mathrm{min} / 1 \cdot 73 \mathrm{~m}^{2}\right)$ & $75 \cdot 0$ & $19 \cdot 0$ & $74 \cdot 0$ & $21 \cdot 0$ \\
\hline BMI $\left(\mathrm{kg} / \mathrm{m}^{2}\right)$ & $24 \cdot 7$ & $3 \cdot 3$ & $25 \cdot 3^{*}$ & $3 \cdot 2$ \\
\hline Height (cm) & $164 \cdot 0$ & $6 \cdot 0$ & $165 \cdot 0$ & $6 \cdot 0$ \\
\hline Weight (kg) & $66 \cdot 5$ & $10 \cdot 3$ & $68 \cdot 8^{*}$ & $10 \cdot 4$ \\
\hline $\mathrm{SBP}(\mathrm{mmHg})$ & 134 & 23 & $131^{*}$ & 22 \\
\hline $\mathrm{DBP}(\mathrm{mmHg})$ & 78 & 13 & 78 & 13 \\
\hline $\mathrm{Hb}(\mathrm{g} / \mathrm{dl})$ & $14 \cdot 6$ & $1 \cdot 4$ & $14 \cdot 7$ & $1 \cdot 5$ \\
\hline Platelets $\left(10^{3} / \mu \mathrm{l}\right)$ & 198 & 55 & 201 & 59 \\
\hline AST (IU/I) & 33 & 30 & $37^{*}$ & 27 \\
\hline ALT (IU/I) & 35 & 52 & 38 & 34 \\
\hline BUN (mg/dl) & 18 & 6 & 19 & 9 \\
\hline Creatinine (mg/dl) & $1 \cdot 1$ & $0 \cdot 4$ & $1 \cdot 1$ & $0 \cdot 7$ \\
\hline Uric acid (mg/dl) & $6 \cdot 5$ & $1 \cdot 5$ & $6 \cdot 4$ & $1 \cdot 5$ \\
\hline Albumin (g/dl) & $4 \cdot 0$ & $0 \cdot 3$ & $4 \cdot 0$ & 0.4 \\
\hline Globulin (g/dl) & $3 \cdot 2$ & 0.5 & $3 \cdot 2$ & 0.5 \\
\hline Cholesterol (mg/dl) & 194 & 39 & 191 & 41 \\
\hline TAG (mg/dl) & 123 & 99 & $166^{*}$ & 259 \\
\hline \multirow[t]{2}{*}{ Fasting blood glucose $(\mathrm{mg} / \mathrm{dl})$} & 112 & 45 & $118^{*}$ & 43 \\
\hline & $n$ & $\%$ & $n$ & $\%$ \\
\hline Chronic kidney disease & 321 & $11 \cdot 3$ & 106 & $24 \cdot 8^{\star}$ \\
\hline \multicolumn{5}{|l|}{ Co-morbidity } \\
\hline Hypertension & 664 & $23 \cdot 4$ & 85 & $19 \cdot 9$ \\
\hline Diabetes & 356 & $12 \cdot 5$ & 50 & $11 \cdot 7$ \\
\hline Hyperlipidaemia & 113 & $4 \cdot 0$ & 14 & $3 \cdot 1$ \\
\hline \multicolumn{5}{|l|}{ Lifestyle } \\
\hline Smoking & 661 & $23 \cdot 3$ & 297 & $69 \cdot 6^{\star}$ \\
\hline Alcohol consumption & 624 & $22 \cdot 0$ & 231 & $54 \cdot 1^{*}$ \\
\hline
\end{tabular}

eGFR, estimated glomerular filtration rate by Modification of Diet in Renal Disease formula; SBP, systolic blood pressure; DBP, diastolic blood pressure; AST, aspartate aminotransferase; ALT, alanine aminotransferase; BUN, blood urea nitrogen.

${ }^{\star} P<0.05 \mathrm{v}$. betel nut $(-)$ in independent $t$ test, Mann-Whitney $U$ test or $\chi^{2}$ test.

betel-nut use. The prevalence of CKD was significantly higher in participants with betel-nut use than in those without $(24 \cdot 8 \% v \cdot 11 \cdot 3 \%, P=0 \cdot 026)$. The demographic data and biochemical characteristics of the entire male study group are shown in Table 1 . As can be seen, participants with CKD were significantly older and had higher SBP, serum BUN, creatinine and uric acid levels than participants without CKD. The prevalence of hypertension, diabetes and betel-nut chewing was significantly higher in CKD participants than non-CKD participants. $\mathrm{Hb}, \mathrm{ALT}$ and albumin levels were significantly lower in participants with CKD than in those without.

The prevalence of CKD was $24 \cdot 8 \%$ (106/427) in betelnut users, which was significantly higher than that $(11 \cdot 3 \%, 321 / 2837)$ of non-users. As shown in Table 2, the participants with betel-nut use were younger and had lower SBP than those without. Serum TAG and fasting blood glucose levels were significantly higher in participants with betel-nut use than those without. In addition, participants with betel-nut use also had higher prevalence of smoking and alcohol usage. The unadjusted OR of
Table 3 Unadjusted odds ratios and 95\% confidence intervals for association of chronic kidney disease with various risk factors in univariate logistic regression analysis: retrospective review of healthcheck records of 3264 men in a hospital-based cross-sectional screening programme, Yunlin County, Taiwan, 2003 to 2006

\begin{tabular}{lccr}
\hline & OR & $95 \% \mathrm{Cl}$ & \multicolumn{1}{c}{$P$} \\
\hline Hypertension & 2.407 & $2.002,2 \cdot 894$ & $<0.001$ \\
Diabetes & 1.332 & $1.046,1.697$ & 0.020 \\
Smoking & 0.736 & $0.606,0.894$ & 0.002 \\
Alcohol consumption & 0.474 & $0.379,0.591$ & $<0.001$ \\
Betel-nut use & 1.310 & $1.033,1.663$ & 0.026 \\
Hypertension + betel-nut use & 2.766 & $1.781,4.297$ & $<0.001$ \\
Diabetes + betel-nut use & 1.993 & $1.104,3.601$ & 0.022 \\
\hline
\end{tabular}

Table 4 Odds ratios and $95 \%$ confidence intervals for association of chronic kidney disease with various risk factors in multivariate logistic regression analysis after adjustment for age, hypertension and diabetes: retrospective review of health-check records of 3264 men in a hospital-based cross-sectional screening programme, Yunlin County, Taiwan, 2003 to 2006

\begin{tabular}{lccc}
\hline & OR & $95 \% \mathrm{Cl}$ & $P$ \\
\hline Betel-nut use & 2.572 & $1.917,3.451$ & $<0.001$ \\
BMI $^{*}$ & 1.008 & $0.979,1.037$ & 0.61 \\
Smoking & 1.007 & $0.799,1.270$ & 0.950 \\
Hyperlipidaemia & 1.055 & $0.651,1.709$ & 0.827 \\
Alcohol consumption & 0.786 & $0.609,1.015$ & 0.064 \\
\hline
\end{tabular}

*OR calculated per $1 \mathrm{~kg} / \mathrm{m}^{2}$ increment.

CKD for traditional risk factors in univariate logistic regression analyses are shown in Table 3. Hypertension and diabetes were associated with increased CKD risk: $\mathrm{OR}=2 \cdot 407(95 \%$ CI $2 \cdot 002,2 \cdot 894, P<0 \cdot 001)$ and $\mathrm{OR}=$ 1.332 (95\% CI $1 \cdot 046,1 \cdot 697, P=0 \cdot 020)$, respectively. For the individuals with hypertension and betel-nut use, the OR for CKD increased to $2 \cdot 766$ (95\% CI $1 \cdot 781,4 \cdot 297$, $P<0 \cdot 001)$. For participants with diabetes and betel-nut use, the OR for CKD was 1.993 (95\% CI $1 \cdot 104,3 \cdot 601$, $P=0 \cdot 022$ ). These findings suggest the synergic effect of betel-nut use on CKD risk in patients with diabetes or hypertension.

Risk factors for CKD including smoking, alcohol consumption, BMI and hyperlipidaemia were taken into consideration in multivariate logistic regression analyses with adjustments for age, hypertension and diabetes. As shown in Table 4, betel-nut use, independent of BMI, hyperlipidaemia smoking or alcohol consumption, was significantly associated with CKD $(P<0 \cdot 001)$. The adjusted OR for betel-nut use was $2 \cdot 572$ (95\% CI $1 \cdot 917$, 3.451), suggesting that betel-nut use may be a more important CKD risk factor than alcohol consumption, smoking, BMI or hyperlipidaemia.

\section{Discussion}

We found that exposure to betel nut was independently associated with CKD in men based on the data of a 
hospital-based health-check programme. This postulate was supported by three findings: (i) participants with a history of betel-nut use had a higher prevalence of CKD than those without; (ii) betel-nut use was independently associated with a $2 \cdot 6$-fold increased risk of CKD after adjustment for age, hypertension and diabetes, and the association was independent of BMI, smoking, alcohol consumption and hyperlipidaemia; and (iii) the use of betel nut increased further the risk for CKD in individuals with diabetes or hypertension.

Betel-nut use, an established risk factor for oropharyngeal malignancy, is associated with hyperglycaemia, obesity and metabolic syndrome ${ }^{(8)}$. Our finding may be explained by several hypotheses. First, the aqueous extract of betel nut can induce breaks in DNA of kidney cells in an animal model ${ }^{(15)}$. A decreased eGFR has been reported for a subject who regularly consumed about 40 betel nuts/ ${ }^{(16)}$. Second, participants with betel-nut use have a high prevalence of smoking $^{(17)}$ and alcohol consumption ${ }^{(18)}$ that are themselves risk factors for CKD. The result of multivariate logistic regression analysis (Table 4) suggested that the influence of betel nut may overpower the influence of smoking and alcohol consumption. Third, betel-nut use is associated with metabolic syndrome and obesity ${ }^{(19,20)}$ which are important risk factors for CKD. It is possible that the use of betel nut is associated with CKD through the influence of metabolic syndrome or obesity ${ }^{(8,21)}$. Betel-nut users had higher serum TAG and fasting blood sugar (Table 2) than non-users; however, the data on HDL and waist circumferences were not available in our study. In view of the effect of CKD on global health, our finding had an important epidemiological implication.

The prevalence of CKD was 20.7\% in our study (agecorrected prevalence $13 \cdot 8 \%$ ), higher than the previous reported prevalence of CKD among the general population in Taiwan ${ }^{(1,22)}$. Participants in the present study were older than the general population so they might be more at risk for CKD. Nevertheless, the prevalence of betel-nut chewing in our study is close to previous community-based cohort data $^{(8,21)}$. In addition, participants with betel-nut use had lower SBP than those without, which may be explained by the peripheral cholinergic effect of betel nut and by the relatively younger age of betel-nut chewers.

In conclusion, our study demonstrates a significant association between betel-nut use and CKD in men and this association is independent of age, hypertension, diabetes, BMI, smoking, alcohol consumption and hyperlipidaemia. More studies are needed to confirm our findings and prospective studies are needed to investigate the effect of cessation of betel-nut use in CKD.

\section{Acknowledgements}

We have no conflicts for this study. We received no funding for this study.
Author contributions: C.-Y.C. - entire text; S.-Y.C., J.-H.L. and W.-C.C. - co-authors of text and editorial revision; I.-M.K. and C.-M.S. - data collection and analysis; W.C and Y.-H.T. - supervision of the study and writing.

\section{References}

1. Kuo HW, Tsai SS, Tiao MM \& Yang CY (2007) Epidemiological features of CKD in Taiwan. Am J Kidney Dis $\mathbf{4 9}$, 46-55.

2. Yang WC, Hwang SJ, Chiang SS, Chen HF \& Tsai ST (2001) The impact of diabetes on economic costs in dialysis patients: experiences in Taiwan. Diabetes Res Clin Pract 54, Suppl. 1, S47-S54.

3. Lysaght MJ (2002) Maintenance dialysis population dynamics: current trends and long-term implications. J Am Soc Nephrol 13, Suppl. 1, S37-S40.

4. National Kidney Foundation (2002) K/DOQI clinical practice guidelines for chronic kidney disease: evaluation, classification, and stratification. Am J Kidney Dis 39, S1-S266.

5. Gupta PC \& Warnakulasuriya S (2002) Global epidemiology of areca nut usage. Addict Biol 7, 77-83.

6. IARC Working Group on the Evaluation of Carcinogenic Risks to Humans (2004) Betel-quid and areca-nut chewing and some areca-nut derived nitrosamines. IARC Monogr Eval Carcinog Risks Hum 85, 1-334.

7. Ho PS, Ko YC, Yang YH, Shieh TY \& Tsai CC (2002) The incidence of oropharyngeal cancer in Taiwan: an endemic betel quid chewing area. J Oral Pathol Med 31, 213-219.

8. Yen AM, Chiu YH, Chen LS, Wu HM, Huang CC, Boucher BJ \& Chen TH (2006) A population-based study of the association between betel-quid chewing and the metabolic syndrome in men. Am J Clin Nutr 83, $1153-1160$.

9. Tseng C-H (2006) Betel nut chewing is independently associated with urinary albumin excretion rate in type 2 diabetic patients. Diabetes Care 29, 462-463.

10. Huang YG, Tseng HM \& Luo JC (2002) Findings of anthropometric and laboratory data from adult health screening under the National Health Insurance plan in Taiwan. Chang Gung Med J 25, 29-38.

11. Hyman DJ \& Pavlik VN (2001) Characteristics of patients with uncontrolled hypertension in the United States. $N$ Engl J Med 345, 479-486.

12. Hwang SJ, Ballantyne CM, Sharrett AR, Smith LC, Davis CE, Gotto AM Jr \& Boerwinkle E (1997) Circulating adhesion molecules VCAM-1, ICAM-1, and E-selectin in carotid atherosclerosis and incident coronary heart disease cases: the Atherosclerosis Risk In Communities (ARIC) study. Circulation 96, 4219-4225.

13. Schachinger V, Britten MB \& Zeiher AM (2000) Prognostic impact of coronary vasodilator dysfunction on adverse long-term outcome of coronary heart disease. Circulation 101, 1899-1906.

14. Gigleux I, Gagnon J, St-Pierre A, Cantin B, Dagenais GR, Meyer F, Despres JP \& Lamarche B (2006) Moderate alcohol consumption is more cardioprotective in men with the metabolic syndrome. J Nutr 136, 3027-3032.

15. Wary KK \& Sharan RN (1988) Aqueous extract of betel-nut of north-east India induces DNA-strand breaks and enhances rate of cell proliferation in vitro. Effects of betel-nut extract in vitro. J Cancer Res Clin Oncol 114, 579-582.

16. Lin S-H, Lin Y-F, Cheema-Dhadli S, Davids MR \& Halperin ML (2002) Hypercalcaemia and metabolic alkalosis with 
betel nut chewing: emphasis on its integrative pathophysiology. Nephrol Dial Transplant 17, 708-714.

17. Stengel B, Tarver-Carr ME, Powe NR, Eberhardt MS \& Brancati FL (2003) Lifestyle factors, obesity and the risk of chronic kidney disease. Epidemiology 14, 479-487.

18. Shankar A, Klein R \& Klein BE (2006) The association among smoking, heavy drinking, and chronic kidney disease. Am J Epidemiol 164, 263-271.

19. Chung FM, Chang DM, Chen MP, Tsai JC, Yang YH, Shieh TY, Shin SJ, Chen TH, Tai TY \& Lee YJ (2006) Areca nut chewing is associated with metabolic syndrome: role of tumor necrosis factor- $\alpha$, leptin, and white blood cell count in betel nut chewing-related metabolic derangements. Diabetes Care 29, 1714.
20. Chen TH, Chiu YH \& Boucher BJ (2006) Transgenerational effects of betel-quid chewing on the development of the metabolic syndrome in the Keelung Community-based Integrated Screening Program. Am J Clin Nutr 83, 688-692.

21. Chang WC, Hsiao CF, Chang HY, Lan TY, Hsiung CA, Shih YT \& Tai TY (2006) Betel nut chewing and other risk factors associated with obesity among Taiwanese male adults. Int J Obes (Lond) 30, 359-363.

22. Hsu CC, Hwang SJ, Wen CP, Chang HY, Chen T, Shiu RS, Horng SS, Chang YK \& Yang WC (2006) High prevalence and low awareness of CKD in Taiwan: a study on the relationship between serum creatinine and awareness from a nationally representative survey. Am J Kidney Dis $\mathbf{4 8}$, $727-738$. 